\title{
Implicações Cardiovasculares em Pacientes Infectados com Covid-19 e a Importância do Isolamento Social para Reduzir a Disseminação da Doença
}

\author{
Cardiovascular Implications in Patients Infected with Covid-19 and the Importance of Social Isolation to \\ Reduce Dissemination of the Disease
}

Juliana Alves Costa, ${ }^{1 \oplus}$ Juliana de Almeida Silveira, ${ }^{1}$ Sara Cristine Marques dos Santos, ${ }^{1 \oplus}$ Patrícia Pereira Nogueira ${ }^{1}$ Universidade de Vassouras - Cardiologia, ${ }^{1}$ Rio de Janeiro, RJ - Brasil

\section{Resumo}

Os sintomas respiratórios, principalmente o desenvolvimento de quadros de síndrome do desconforto respiratório agudo grave, dominam a discussão e as preocupações iniciais da população e dos profissionais de saúde. Entretanto, o sistema cardiovascular é bastante afetado por essas condições e, muitas vezes, é o responsável por complicações e mortalidade desses pacientes. Com o objetivo de mostrar as implicações cardiovasculares em pacientes infectados pela COVID-19 e a importância do isolamento social como alternativa de frear a disseminação da doença, foi realizada uma revisão da literatura com base em 37 artigos, nos idiomas inglês, português e espanhol, disponíveis na plataforma Scielo e PubMed. Os resultados mostraram que complicações cardíacas associadas à infecção pela COVID-19 são semelhantes às produzidas por: síndrome respiratória aguda grave (SARS), síndrome respiratória do Médio Oriente (MERS) e influenza. Contudo, a COVID-19 apresenta uma contaminação muito maior e mais rápida e, ao contrário da gripe por influenza, ainda não existe vacina disponível ou tratamento. Diante disso, o isolamento social passa a ser uma ferramenta que pode reduzir e achatar a curva de casos incidentes e assim preservar as pessoas que se enquadrem no grupo de risco, diminuindo as chances de quadros graves da doença, possíveis óbitos e o colapso no sistema de saúde do país.

\section{Introdução}

O coronavírus se trata de um vírus pertencente à família Coronaviridae, causadores de uma gripe simples até doenças que podem causar risco maior à saúde da população. O

\section{Palavras-chave}

Coronavirus; COVID-19; Infecções por Coronavirus/ prevenção e controle; Isolamento Social; Disseminação da Doença.

\footnotetext{
Correspondência: Juliana Alves Alves Costa •

Universidade de Vassouras - Cardiologia

Av. Expedicionário Osvaldo de Almeida Ramos, 280. CEP 27700-000,

Centro, Vassouras, RJ - Brasil

E-mail: jujuh.ac@hotmail.com

Artigo recebido em 24/03/2020, revisado em 15/04/2020,

aceito em 15/04/2020
}

novo coronavírus, causador da pandemia em 2020, recebeu a denominação SARS-CoV-2 pela Organização Mundial da Saúde (OMS), e a doença que ele provoca tem a denominação COVID-19. ${ }^{1}$ Primeiramente foi detectado em dezembro de 2019 em Wuhan, na China. No entanto, em virtude de seu alto poder de disseminação, diversos países confirmaram a presença de casos alóctones em meados de janeiro de 2020. No Brasil, confirmou-se o primeiro caso no dia 26 de fevereiro de $2020 .^{2,3}$

Até o aparecimento do SARS-CoV-2, estavam descritas duas outras epidemias causadas por coronavírus: o SARSCoV-1, causador da síndrome respiratória aguda grave (SARS), em 2002; e MERS-CoV, causador da síndrome respiratória do Médio Oriente (MERS), em 2012.4 A fisiopatologia do SARS-CoV-2 demostrou-se semelhante à do SARS-CoV-1, pois apresentam lesões pulmonares agudas devido à inflamação agressiva iniciada pela replicação viral. A infecção por SARSCoV-2 pode causar aumento da secreção de interleucinas pró-inflamatórias e de interferon-gama (IFN- $\gamma$ ) que provocam as lesões pulmonares. ${ }^{5}$

O Brasil, assim como outros países, passa pelo processo de transição demográfica que tem como principal efeito o envelhecimento da população. Assim, as doenças do sistema circulatório aparecem como principal causa de mortalidade da população. Associando essa informação aos estudos recentes das implicações cardiovasculares e seu agravamento pelo SARS-CoV-2, fica evidente que medidas de prevenção e controle que diminuam riscos de contaminação e infecção são importantes ferramentas na diminuição de casos graves da doença e eventuais óbitos.

O presente artigo tem como objetivo relacionar a atual pandemia de COVID-19 com as implicações cardiovasculares, mostrando a importância do isolamento social como medida de prevenção e controle de disseminação da doença e preservação do Sistema de Saúde do país.

\section{Material e métodos}

Revisão da literatura com base em 37 artigos, nos idiomas inglês, português e espanhol, disponíveis na plataforma Scielo e PubMed, referentes as implicações cardiovasculares nos pacientes infectados pelo covid-19, importância do isolamento social como medida de prevenção e controle de disseminação da doença e preservação do sistema de saúde do país.

DOI: https://doi.org/10.36660/abc.20200243 


\section{Panorama geral da COVID-19}

A pandemia da COVID-19, assim como as epidemias prévias de outros coronavírus (SARS e MERS) e a pandemia de 2009 (H1N1), traz consequências graves nos modelos de saúde, econômicos e sociais de toda a população mundial.

A transição demográfica, apesar de ocorrer de forma diferenciada entre os países, de modo geral, caracteriza-se pelo aumento da população idosa em comparação às demais faixas etárias, pois cresce cerca de $4 \%$ ao ano. Fatores como diminuição da fecundidade, redução da mortalidade infantil e da mortalidade geral, melhorias na atenção à saúde da população, desenvolvimento tecnológico no tocante ao diagnóstico e tratamento das doenças também corroboram para o quadro demográfico atual. ${ }^{6}$

Concomitante ao aumento do número de idosos, ocorre uma transição epidemiológica, com crescimento da proporção das doenças no aparelho circulatório, diabetes melito, neoplasias, agravos por causas externas e doenças do aparelho respiratório. ${ }^{7}$ Estudos indicam que uma maior frequência de comorbidades está comumente relacionada à idade mais avançada.

A taxa de mortalidade da COVID-19 pode ser nove vezes maior entre pessoas com alguma doença crônica, quando comparada à de pacientes sem patologia preexistente. Dados disponibilizados pela Organização Mundial da Saúde (OMS) em fevereiro demonstram que no grupo de infectados sem comorbidade apenas 1,4\% morreu. Já entre os pacientes com alguma doença cardiovascular, por exemplo, o índice chegou a 13,2\%. Considerando todos os pacientes infectados, a letalidade foi de 3,8\%, mas vale ressaltar que, em função do andamento da pandemia, novos dados estatísticos vêm sendo adicionados aos estudos.

A forma severa da doença foi observada nos pacientes mais velhos ${ }^{8,9}$ que apresentavam um número mais significativo de condições comórbidas comparados aos pacientes não graves. Esses resultados sugerem que idade e comorbidades associadas podem ser um dos fatores de risco para pacientes críticos. Além disso, idosos e pacientes imunossuprimidos podem manifestar sintomas atípicos e outras maneiras de apresentação, incluindo pneumonia leve, moderada e grave, e em casos mais graves, síndrome respiratória aguda grave, sepse, choque séptico e morte. ${ }^{4}$

Em um relato de caso de 138 pacientes hospitalizados com COVID-19, 16,7\% dos pacientes desenvolveram arritmia e $7,2 \%$ sofreram lesão cardíaca aguda, além de outras complicações relacionadas com a COVID-19. Relatórios publicados indicam casos de insuficiência cardíaca de início agudo, infarto do miocárdio, miocardite e parada cardíaca. ${ }^{10}$ Além disso, foram encontrados casos de dano miocárdico, com aumento de troponina I, dano cardíaco agudo, choque e arritmia. ${ }^{11,12}$

$\mathrm{Na}$ fase aguda dos quadros virais graves, não somente da COVID-19, mas também em outras coronoviroses, o paciente pode apresentar taquicardia, hipotensão, bradicardia, arritmias e morte súbita. Alterações eletrocardiográficas e aumento de troponina sinalizam acometimento miocárdico na forma de miocardite. ${ }^{11,12}$
Estudos de coortes publicadas até o momento mostram taxas de insuficiência cardíaca aguda, choque e arritmia de $7,2 \%, 8,7 \%$ e $16,7 \%$, respectivamente. O acometimento cardiovascular decorre devido a um descompasso entre o aumento da demanda metabólica/inflamatória desencadeado pelo vírus e uma reserva cardíaca reduzida. O estado inflamatório torna o ambiente mais propenso a fenômenos trombóticos. Sendo assim, a recomendação tem sido de que as medicações de uso crônico dos pacientes sejam mantidas, sendo a sua retirada/substituição avaliada em nível individual e de acordo com as diretrizes vigentes até o momento. Vale ressaltar que novas recomendações podem surgir à medida que saem novos trabalhos em andamento. ${ }^{13,14}$

As doenças crônicas, tais como hipertensão, diabetes, doenças do sistema respiratório, doenças cardiovasculares e suas condições de suscetibilidade, compartilham com as doenças infecciosas alguns estados padronizados, como o estado pró-inflamatório e a atenuação da resposta imune inata. O diabetes, por exemplo, ocorre, em parte, porque o acúmulo de células imunes inatas ativadas nos tecidos metabólicos leva à liberação de mediadores inflamatórios, especialmente IL-1 $\beta$ e TNF $\alpha$, que promovem resistência à insulina e danos às células $\beta .^{15}$ Além disso, os distúrbios metabólicos podem levar à depressão da função imunológica, prejudicando a função dos macrófagos e linfócitos, ${ }^{16}$ o que pode tornar os indivíduos mais suscetíveis a complicações e agravos da COVID-19. ${ }^{9}$

Muitos dos pacientes mais velhos que ficam gravemente doentes têm evidências de doenças subjacentes, tais como doença cardiovascular, doença hepática, doença renal ou tumores malignos. ${ }^{17-19}$ Esses pacientes geralmente morrem de suas comorbidades originais, portanto, a avaliação precisa de todas as comorbidades originais dos indivíduos com COVID-19 deve ser rigorosamente analisada e considerada no plano terapêutico individualizado.

Outros trabalhos acrescentam que a insuficiência respiratória agravada pelo SARS-CoV-2 ocorre em virtude de danos alveolares maciços. Esse vírus é capaz de infectar células epiteliais respiratórias humanas por meio de uma interação entre a proteína S viral e o receptor da enzima 2 de conversão da angiotensina nas células humanas. Embora, na literatura, haja evidências de que a presença de infecções pulmonares graves possa afetar o prognóstico a longo prazo dos indivíduos cardiopatas, não existem dados que confirmem que pacientes recuperados da infecção por COVID-19 irão experimentar efeitos a longo prazo. ${ }^{20,21}$

Assim, não apenas capaz de causar pneumonia, a COVID-19 também pode provocar danos a outros órgãos, e os pacientes acabam morrendo por insuficiência de múltiplos órgãos, choque, síndrome do desconforto respiratório agudo, insuficiência cardíaca, arritmias e insuficiência renal. ${ }^{22}$ As possíveis lesões de múltiplos órgãos e sua proteção e prevenção devem ser monitoradas no tratamento da COVID-19. ${ }^{23}$ Nesses pacientes críticos, as medidas de proteção necessárias incluem ventilação mecânica, glicocorticoides, antivirais, tratamentos sintomáticos e terapia antichoque.

Outro fator importante seria a maneira de estimar a capacidade de transmissibilidade de um vírus por meio do 
cálculo de seu número reprodutivo (R0), o que representa uma medida de sua taxa de ataque, ou seja, traduz o número de infecções secundárias que ocorrem a partir de um indivíduo infectado em uma população suscetível. Estudos preliminares apontavam que este novo coronavírus, responsável pela COVID-19, estaria associado a taxas de R0 de 1,5 a 3,5, sendo os dados mais recentes sugerindo um R0 de 4,08 (i. e., para cada caso, em média, haveria quatro novos indivíduos infectados). ${ }^{7}$

Por apresentar alto potencial de disseminação, ${ }^{1}$ e sabendo que se trata de um vírus RNA, envelopado e com contaminação por gotículas respiratórias ou contato, as medidas de higiene devem ser reforçadas e colocadas em prática. Estas são: lavar as mãos com água e sabão para destruir a estrutura morfológica do vírus, usar álcool 70\%, cobrir a boca ao tossir ou espirrar para evitar que as partículas virais se disseminem pelo ambiente, evitar aglomerações e manter-se em local bem ventilado.,

De acordo com a literatura, o período médio de incubação por coronavírus é de 5 dias, com intervalos que chegam a 12 dias. Dados preliminares do SARS-CoV-2 sugerem que a transmissão possa ocorrer mesmo sem o aparecimento de sinais e sintomas. ${ }^{4,5}$

Quando não há complicações, os sintomas apresentados consistem em febre, tosse seca e sensação de cansaço, podendo haver ocorrência de coriza e congestão nasal, dor de garganta e diarreia. Além disso, grande parte dos infectados é assintomática (cerca de $80 \%$ ) e se recupera sem a necessidade de tratamento especial, enquanto $1 / 6$ dos pacientes pode evoluir de forma grave, cursando com dificuldade para respirar. ${ }^{24,25}$
Diante da situação atual, é de extrema importância que a população se conscientize e faça isolamento domiciliar, apresentando-se sintomática ou não, com o objetivo de diminuir o número de pessoas contaminadas e atrasar a transmissão comunitária disseminada, para que o sistema de saúde público consiga atender a todos. ${ }^{26}$ Caso contrário, o crescimento exponencial da doença poderá colapsar este sistema, levando a óbito aqueles mais fragilizados. Já as pessoas que tiverem a necessidade de circular em locais públicos, por motivo de trabalho ou força maior, devem adotar medidas preventivas. ${ }^{27}$

Desacelerar a disseminação do vírus para que o número de casos se espalhe ao longo do tempo em vez de haver picos no início é uma das formas de achatar a curva epidêmica e evitar que o sistema púbico de saúde entre em colapso e, como consequência, muitas pessoas acabem indo a óbito (Figura 1). Uma transmissão controlada da doença reduz a pressão sobre o sistema de saúde e aumenta a capacidade de cuidados não apenas para os pacientes contaminados por coronavírus, mas também para aqueles que necessitam de atendimento médico por outras enfermidades.

O isolamento domiciliar é uma maneira de permanecer em casa tomando alguns cuidados que diminuem o risco de transmitir infecções respiratórias, como a provocada pelo coronavírus (COVID-19). Esses cuidados especiais impedem que o contato das secreções respiratórias de uma pessoa que possa estar infectada pelo coronavírus entrem em contato com outras. ${ }^{19}$ Devem ficar nessa circunstância pessoas que positivaram no teste para o referido vírus ou que estão sob suspeita. O ideal é que o indivíduo fique sozinho em um

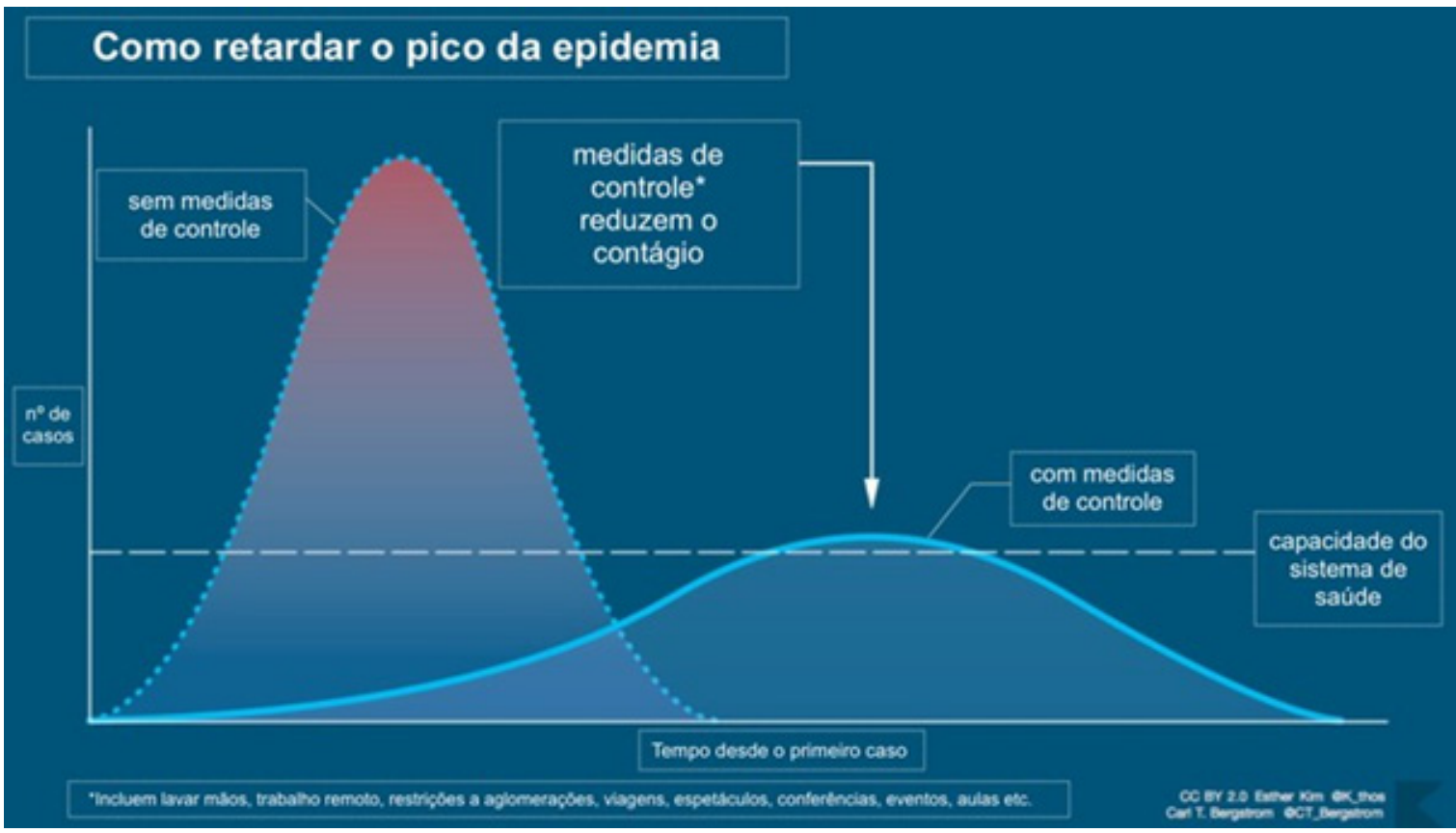

Figura 1 - Curva de disseminação do vírus. 
quarto, ou em um cômodo da casa adaptado como quarto, se possível com um banheiro privativo ${ }^{28,29}$ As portas do quarto devem ficar fechadas o tempo todo, mas as janelas devem ficar abertas para que o ambiente fique bem ventilado. $\mathrm{O}$ paciente só deve sair desse cômodo isolado em caso de necessidade..$^{30-32}$

Diante disso, o isolamento social e as medidas de prevenção são necessários para evitar, principalmente, que os idosos se infectem e venham a agravar doenças preexistentes, complicando o quadro de saúde e levando a sinais e sintomas que muitas vezes podem ser fatais. ${ }^{33,34}$ Quando uma doença se espalha de maneira rápida, os serviços ficam superlotados, não há leitos, máscaras, médicos, respiradores e outros equipamentos suficientes para quem precisa, e isso não só para a COVID-19, mas para qualquer outra enfermidade que requeira que o paciente procure o sistema de saúde. É preciso evitar que o sistema entre em colapso. ${ }^{35}$

\section{Considerações finais}

Há um consenso entre os autores de que o grupo de maior risco para desenvolver a forma mais grave, podendo evoluir para óbito, inclui idosos e indivíduos que apresentem as comorbidades mais prevalentes, dentre elas as doenças cardiovasculares. ${ }^{36}$

O crucial não é a gravidade da doença em si, mas a capacidade de dar atenção a todos os infectados no momento em que eles precisarem. Quanto mais se achata a curva de transmissão ao longo do tempo, menor a sobrecarga no sistema de saúde e maior a probabilidade de que ele dê conta da demanda epidêmica
- o que evidencia a importância do isolamento social como medida de prevenção e controle de disseminação da doença e preservação do sistema de saúde do país.

\section{Contribuição dos autores}

Concepção e desenho da pesquisa: Costa JÁ; Obtenção de dados e Análise e interpretação dos dados: Costa JA, Silveira JA, Santos SCM; Redação do manuscrito: Costa JA, Silveira JA, Costa JA, Silveira JA, Santos SCM, Nogueira PP; Revisão crítica do manuscrito quanto ao conteúdo intelectual importante: Nogueira PP.

\section{Potencial conflito de interesses}

Declaro não haver conflito de interesses pertinentes.

\section{Fontes de financiamento}

O presente estudo não teve fontes de financiamento externas.

\section{Vinculação acadêmica}

Não há vinculação deste estudo a programas de pósgraduação.

\section{Aprovação ética e consentimento informado}

Este artigo não contém estudos com humanos ou animais realizados por nenhum dos autores.

\section{Referências}

1. Lana RM, Coelho FC, Gomes MFC, Cruz OG, Bastos LS, Villela DAM, et al. The novel coronavirus (SARS-CoV-2) emergency and the role of timely and effective national health surveillance. Cad Saúde Pública. 2020;36(3):e00019620.

2. Ministério da Saúde. Brasil confirma primeiro caso da doença. Rio de Janeiro, DF: Ministério da Saúde; 2020. [citado 22 mar. 2020]. Disponível em: <https://www.saude.gov.br/noticias/agencia-saude/46435-brasilconfirma-primeiro-caso-de-novo-coronavirus $>$.

3. Diagnósticos da América SA. Coronavírus: o que é, sintomas e como se prevenir da COVID-19. [citado 22 mar. 2020]. Disponível em: <https:// dasa.com.br/coronavirus $>$.

4. World Health Organization. Coronavirus disease 2019 (COVID-19) situation report - 52.Geneva: WHO; 2020. [citado 22 mar. 2020]. Disponível em: https://www.who.int/docs/default-source/coronaviruse/20200312-sitrep52-covid-19. pdf?sfvrsn=e2bfc9c0 2 .

5. De Carvalho, Aroldo Prohmann. Novo coronavírus (COVID-19). Brasil, fevereiro de 2020, v.14; Disponível em: < https://www.sbp.com.br/ fileadmin/user_upload/22340d-DocCientifico___Novo_coronavirus.pdf > . Acesso em 23 de Março de 2020

6. Lebrão ML. O envelhecimento no Brasil: aspectos da transição demográfica e epidemiológica. Saúde Coletiva. 2007;4(17):135-40.

7. Abbott PA, Barbosa SFF. Using information technology and social mobilization to combat disease.. Acta Paul Enferm .2015;28(1):III-V.
8. Wang T, Du Z, Zhu F, Cao Z, An Y, Gao Y, et al. Comorbidities and multi-organ injuries in the treatment of COVID-19. Lancet. 2020;395(10228):e52.

9. Badawi A, Ryoo SG. Prevalence of comorbidities in the Middle East respiratory syndrome coronavirus (MERS-CoV): a systematic review and meta-analysis. Int J Infect Dis. 2016 Aug;49:129-33.

10. Agência de Notícias da AIDS (BR). Coronavírus é desafio para saúde pública, mas pouco preocupante em nível individual; 2020; [citado 23 mar. 2020]. Disponível em: <https://agenciaaids.com.br/noticia/coronavirus-e-desafiopara-saude-publica-mas-pouco-preocupante-em-nivel-individual/> .

11. del Rio C, Malani PN. COVID-19-New insights on a rapidly changing epidemic. JAMA. 2020;323(14):1339-40.

12. Wu C, Chen X, Cai Y, Xia J, Zhou X, Xu S, et al. Risk factors associated with acute respiratory distress syndrome and death in patients with coronavirus disease 2019 pneumonia in Wuhan, China. JAMA Intern Med. 2020 Mar 13. [Epub ahead of print]

13. Sociedade Brasileira de Reumatologia. Atualização das recomendações para os profissionais de saúde e pacientes com doenças inflamatórias imunomediadas: reumatológicas, dermatológicas e gastrointestinais, frente à infecção pelo 2019-nCoV (17/03/2020). [citado 23 mar. 2020]. Disponível em: http://www.sbd.org.br/mm/cms/2020/03/22/atualizacao-covid-19.pdf.

14. Yang J, Zheng Y, Gou X, Pu K, Chen Z, Guo Q, et al. Prevalence of comorbidities in the novel Wuhan coronavirus (COVID-19) infection: a systematic review and meta-analysis. Int J Infect Dis. 2020 Mar 12; pii:S1201-9712(20)30136-3. [Epub ahead of print] 
15. Odegaard JI, Chawla A. Connecting type 1 and type 2 diabetes through innate immunity. Cold Spring Harb Perspect Med. 2012;2(3):a007724.

16. Dooley KE, Chaisson RE. Tuberculosis and diabetes mellitus: convergence of two epidemics.. Lancet Infect Dis. 2009;9(12):737-46.

17. BBC News. Coronavirus disease named Covid-19. [citado 23 mar. 2020]. Disponível em: https://www.bbc.co.uk/news/world-asia-china-51466362.

18. Jiang S, Xia S, Ying T, Lu L. A novel coronavirus (2019-nCoV) causing pneumonia-associated respiratory syndrome. Cell Mol Immunol. 2020 Feb 5. [Epub ahead of print].

19. Wang D, Hu B, Hu C, Zhu F, Liu X, Zhang J, et al.Clinical characteristics of 138 hospitalized patients with 2019 novel coronavirus-infected pneumonia in Wuhan, China. JAMA. 2020 Feb 7. [Epub ahead of print].

20. Assiri A, McGeer A, Perl TM, Price CS, Al Rabeeah AA, Cummings DA, et al. Hospital outbreak of Middle East respiratory syndrome coronavirus. N Engl Jed. 2013;369(5):407-16

21. Smith SC, Ladenson JH, Mason JW, Jaffe AS. Elevations of cardiac troponin I associated with myocarditis. Experimental and clinical correlates. Circulation. 1997; 95(1):163-8.

22. Huang C, Wang Y, Li X, Ren L, Zhao J, Hu Y, et al. Clinical features of patients infected with 2019 novel coronavirus in Wuhan, China. Lancet. 2020;395(10223):497-506.

23. Chen N, Zhou M, Dong X, Qu J, Gong F, Han Y, et al. Epidemiological and clinical characteristics of 99 cases of 2019 novel coronavirus pneumonia in Wuhan, China: a descriptive study. Lancet. 2020;395(10223):507-13.

24. Dominguez AN, Martín AV. COVID-19. Punto de vista del cardiólogo. Rev Cubana Cardiol. 2020;26(1):1-5.

25. Brasil.Ministério da Saúde. Sobre a doença. Rio de Janeiro, DF:; 2020. [citado 22 mar. 2020]. Disponível em: https://coronavirus.saude.gov.br/ sobre-a-doenca\#transmissao $>$.

26. American College of Cardiology. ACC Clinical Bulletin COVID-19 Clinical Guidance For the Cardiovascular Care Team; 2020.
27. Xiong TY, Redwood S, Prendergast B, Chen M. Coronaviruses and the cardiovascular system: acute and long-term implications. Eur Heart J. 2020 Mar 18;pii:ehaa231. [Epub ahead of print].

28. Poder Judiciário. Tribunal Regional do Trabalho 11르 Região, Ato 15/2020/ SGP. Estabelece medidas temporárias de prevenção ao contagio pelo novo coronavirus (COVl-19) Internet. [Citado em 20 Mar 2020] Disponível em: portal.trt11.jus.br

29. Glasser JW, Hupert N, McCauley MM, Hatchett R. Modeling and public health emergency responses: lessons from SARS. Epidemics. 2011;3(1):32-7.

30. Zhang JJ, Dong X, Cao YY, Yuan YD, Yang YB, Yan YQ, et al. Clinical characteristics of 140 patients infected with SARS-CoV-2 in Wuhan, China. Allergy. 2020 Feb 19. [Epub ahead of print].

31. Huang C, Wang Y, Li X, Ren L, Zhao J, Hu Y, et al. Clinical features of patients infected with 2019 novel coronavirus in Wuhan, China. Lancet. 2020;395(10223):497-506.

32. Kuiken T, Fouchier RAM, Schutten M, Rimmelzwaan GF, van Amerongen G, van Riel D, etal. Newly discovered coronavirus as the primary cause of severe acute respiratory syndrome. Lancet. 2003;362(9380):263-70.

33. Fu Y, Cheng Y, Wu Y. Understanding SARS-CoV-2-mediated inflammatory responses: from mechanisms to potential therapeutic tools. Virol. Sin. 2020 Mar 3. [Epub ahead of print].

34. Guimarães HP, Damasceno MC, Braga MA, Schubert DUC, Santana JCB Freitas APR, et al. Coronavírus e medicina de emergência: recomendações para o atendimento inicial do médico emergencista pela Associação Brasileira de Medicina de Emergência (ABRAMEDE). [citado 23 mar. 2020]. Disponível em: https://www.amib.org.br/fileadmin/user_upload/POSICIONAMENTO_ ABRAMEDE_-_CORONAVIRUS_-_03-_10032020.pdf.

35. Li B, Yang J, Zhao F, Zhi L, Wang X, Liu L, et al. Prevalence and impact of cardiovascular metabolic diseases on COVID-19 in China. Clin Res Cardiol. 2020 Mar 11. [Epub ahead of print].

36. Chinese Center for Disease Control and Prevention. The epidemiological characteristics of an outbreak of 2019 novel coronavirus diseases (COVID-19) in China. Zhonghua Liu Xing Bing Xue Za Zhi. 2020:41(2):145-51. 